OPEN ACCESS

Edited by:

Carolina Romero,

University of Salamanca, Spain

Reviewed by:

Lei Wang,

Jilin University, China

Ye Dai,

Shanghai University, China

*Correspondence:

Chen Cheng

drccheng@sdnu.edu.cn

Specialty section: This article was submitted to

Optics and Photonics,

a section of the journal

Frontiers in Physics

Received: 23 August 2021 Accepted: 29 December 2021

Published: 20 January 2022

Citation:

Zhou Y, Cheng C, Hu L and Chen F (2022) Guided-Wave Up-Conversion Luminescence in $\mathrm{Er}^{3+} / \mathrm{Yb}^{3+}$ Co-doped Phosphate Glass Waveguide Produced by Direct Femtosecond

Laser Writing

Front. Phys. 9:763377.

doi: 10.3389/fphy.2021.763377

\section{Guided-Wave Up-Conversion} Luminescence in $\mathrm{Er}^{3+} / \mathrm{Yb}^{3+}$ Co-doped Phosphate Glass Waveguide Produced by Direct Femtosecond Laser Writing

\author{
Yuxiang Zhou ${ }^{1}$, Chen Cheng ${ }^{1 *}$, Lili Hu ${ }^{2}$ and Feng Chen ${ }^{3}$ \\ ${ }^{1}$ School of Physics and Electronics, Shandong Normal University, Jinan, China, ${ }^{2}$ Shanghai Institute of Optics and Fine Mechanics, \\ Chinese Academy of Sciences, Shanghai, China, ${ }^{3}$ School of Physics, Shandong University, Jinan, China
}

We report on guided-wave up-conversion luminescence in femtosecond laser writing cladding waveguides in $\mathrm{Er}^{3+} \mathrm{Mb}^{3+}$ co-doped phosphate glass. The waveguides were fabricated with 30- $\mu \mathrm{m}$ - and 100- $\mu \mathrm{m}$-diameter of the guiding cores. The guiding properties of waveguides have been investigated at $633 \mathrm{~nm}$ by end face coupling of free space light and physical contact of fibers. The experimental and calculated results of propagating modal profiles and losses have proved favorable performances suitable to Gaussian mode field and multi-mode applications. Under the optical pump laser at $980 \mathrm{~nm}$, the guidedwave up-conversion luminescence at visible light range has been realized through the waveguides.

Keywords: optical waveguides, rare-earth-ion doped glass materials, up-conversion, femtosecond laser micromachining, integrated optical device

\section{INTRODUCTION}

Rare-earth-doped glasses are widely applied in optical communications, optical sensing, imaging, etc., benefiting for their outstanding material properties including high ions solubility, prominent nonlinear effects, and adjustable refractive indices $[1,2]$. Many kinds of glasses, such as silicate glass, chalcogenide glass, phosphate glass, tellurite glass, and fluoride glass were successfully used to fabricate active integrated devices and lasers, by doping rare-earth ions [3-5]. In these glasses, the silicate as well as phosphate glasses are most commonly used for preparing fibers operating around $1 \mu \mathrm{m}$. The phosphate glass can realize a high concentration up to above $20 \mathrm{wt} \%$, contributing to extremely enhance the pump absorption efficiency and thus shorten the fiber length. Thus far, $\mathrm{Nd}^{3+}$ ion doped, $\mathrm{Er}^{3+}$ ion glass and $\mathrm{Yb}^{3+}$ ion doped phosphate glass have been developed in fields of laser fusion, laser weapon, laser ranging, optical communication waveguide amplifier and ultrashort pulse laser $[6,7] . \mathrm{Er}^{3+}$ ion has been regarded as the most efficient candidate to generate up-conversion luminescence due to its appropriate electronic structures [8]. The optical materials co-doped with erbium $\left(\mathrm{Er}^{3+}\right)$ and ytterbium ions $\left(\mathrm{Yb}^{3+}\right)$ exist energy transfer between $\mathrm{Er}^{3+}$ and $\mathrm{Yb}^{3+}$, which provides a straightforward way to combine the $\mathrm{Yb}^{3+}$ ion's simple energy level scheme with the $\mathrm{Er}^{3+}$ ion's electronic states, for low-cost pumping at $980 \mathrm{~nm}$ [9]. Due to a few different mechanisms of $\mathrm{Er}^{3+}$ ions including exited-state absorption and the photon avalanche effect, these materials can be excited the up-conversion luminescence that the wavelength of emission light is shorter than the excitation. Currently, there are great interests in luminescent materials for efficient up-conversion from near 
infrared to visible emission, which is applied in extensively fields of super-resolution imaging, medical diagnoses, sensors [10-12]. Phosphate glasses, mainly composed of $\mathrm{P}_{2} \mathrm{O}_{5}$ instead of $\mathrm{SiO}_{2}$ in silica glasses, can be beneficial over silica glasses with low transform temperature, making the materials to be suffered from heat induced end-face damage at a relative high output power, commonly employed for optical integrations [13, 14].

Waveguides, as a fundamental component in optical integrated circuits, have been achieved for a number of photonic applications in rare-earth-ion doped materials. Benefitting from compact geometry of waveguides and combination of versatile bulk features, miniaturized devices on waveguide platforms can be fabricated with high density, low optical thresholds, on-chip sizes, etc. Optical waveguide is able to localize the propagation of light field within the space at micron scale, thus increase the light density considerably with respect to that in the bulk material. Optical waveguides can be fabricated in many ways, such as ion implantation proton exchange, thermal ion diffusion, thin film deposition, femtosecond laser processing, etc., [15-19]. As a new technology of fabricating waveguide, femtosecond laser can be focused in the material to change the refractive index distribution of the material through a few nonlinear effects to form a waveguide structure [20-23]. Femtosecond laser is often used to prepare optical waveguides in rare-earth-doped materials. In recent years, several phenomenon and applications have been discovered by using femtosecond laser for optical waveguide manufacturing in rareearth-doped glass materials, including planar and channel waveguides [24-26]. In 2016, D.G. Lancaster et al. reported an $\mathrm{Er}^{3+} \mathrm{Yb}^{3+}$ co-doped waveguide laser fabricated by fs laser writing [27]. According to refractive index changes of writing track in all optical materials, laser-writing configuration can be categorized into positive change and negative change. Different from positive changes (so-called Type I with directly refractive index increasing, Type II with laser induced stress change), negative changes are often used to make depressed cladding waveguides (Type III). This type of waveguide can be arbitrarily controlled its cross-section size in the range of ten microns to hundreds of microns [28-30]. In 2018, S.N.C. Santos et al demonstrated the fabrication of 3D Type I waveguides in barium borate glass codoping with $\mathrm{Dy}^{3+}$ and $\mathrm{Eu}^{3+}$ ions for the visible band applications [31]. In 2021, M. Khalid et al showed the femtosecond laser written Type II waveguides in $\mathrm{Yb}$ doped glass for $1 \mu \mathrm{m}$ laser applications [32]. Compared with Type I and II, depressed cladding waveguides commonly are considered to possess better performance in fiber-to-waveguide coupling with adjustable diameter of the end-face cross-section [15, 28-30].

In this work, we have achieved for depressed cladding waveguides with two different cross-section sizes by femtosecond laser writing in $\mathrm{Er}^{3+}$ and $\mathrm{Yb}^{3+}$ co-doped phosphate glass. the up-conversion luminescence of glass waveguides under visible light was studied under NIR optical pumping. Through the end coupling system, we study the waveguide characteristics, transmission mode and transmission loss of the waveguide at $633 \mathrm{~nm}$. Under pump laser at $980 \mathrm{~nm}$, we successfully realized the guidedwave up-conversion luminescence at visible light range

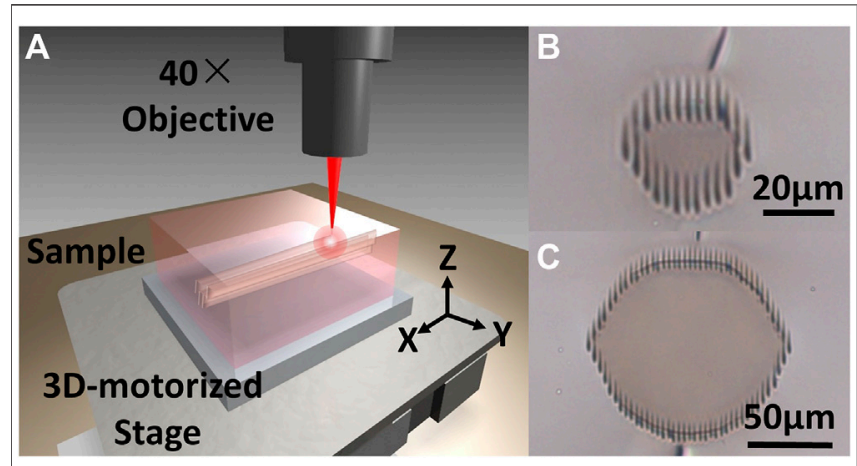

FIGURE 1 | (A) Schematic of the direct femtosecond laser writing process. (B,C) Optical microscope images of the cross sections of depressed cladding waveguides with diameters of 30 and $100 \mu \mathrm{m}$, respectively.

through the waveguides and discussed the possible upconversion mechanism based on the energy match situation dependency on pumping power and the $\mathrm{Er}^{3+}$ and $\mathrm{Yb}^{3+}$ ion energy level diagram.

\section{EXPERIMENTS IN DETAILS}

The phosphate glass for waveguide fabrication is a mature commercial glass made by Shanghai Institute of Optics and Fine Mechanics, Chinese Academy of Science, called WM13. The glass has compositions: $\mathrm{P}_{2} \mathrm{O}_{5}-\mathrm{Na}_{2} \mathrm{O}-\mathrm{BaO}-\mathrm{Al}_{2} \mathrm{O}_{3}-\mathrm{Er}_{2} \mathrm{O}_{3}$ $\mathrm{Yb}_{2} \mathrm{O}_{3}$, with 1.5wt.\% $\mathrm{Er}_{2} \mathrm{O}_{3}$ and 4.5 wt. $\% \mathrm{Yb}_{2} \mathrm{O}_{3}$, which has been cut into dimensions of $10(\mathrm{x}) 10(\mathrm{y}) \times 2(\mathrm{z}) \mathrm{mm}^{3}$ and optically polished. The cladding waveguides with circular boundaries were produced by using a home-made laser fabricating facility. The femtosecond laser system (amplified Ti: Sapphire, Spitfire, Spectra Physics, United States), with $120 \mathrm{fs}$ duration, $800 \mathrm{~nm}$ central wavelength, and $1 \mathrm{kHz}$ repetition rate, was used to generate linearly polarized laser to fabricate waveguides. The laser irradiation was controlled with a mechanical shutter. To control the shape of the beam and the energy on sample, a neutral density filter was placed before the focusing objective lens. The $\times 40$ focusing microscope objective (Numerical Aperture N.A. $=0.40$ ) was used to focus the laser beam at the depth of $\sim 150 \mu \mathrm{m}$. And the phosphate glass sample was inserted on the XYZ translation stage with a constant moving velocity of $0.5 \mathrm{~mm} / \mathrm{s}$ (the schematic plot shown in Figure Figure1A). The procedure was repeated at different depths and positions of the sample following the desired circular geometry. As result, two tubular structures containing a chain of parallel tracks were inscribed in the phosphate crystal by the fslaser. The two depressed cladding waveguides were produced to be 30- and 100- $\mu \mathrm{m}$-diameter with a pulse energy of $\sim 0.42 \mu \mathrm{J}$, shown in Figures 1B,C. The careful choice of the irradiation parameters (scanning velocity and pulse energy) avoided the formation of cracks inside the crystal and allowed the generation of cladding waveguides.

The guidance measurement was performed through an end-face coupling platform. The components in the platform 


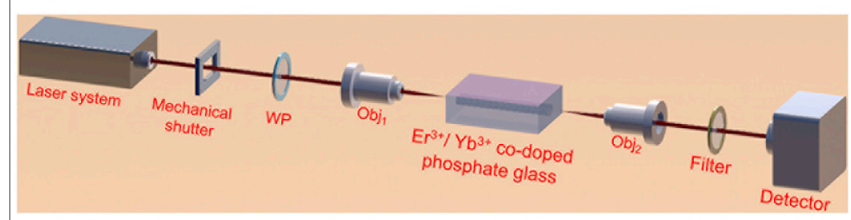

FIGURE 2| The schematic plot of the end-face coupling system using a laser source at $632.8 \mathrm{~nm}$
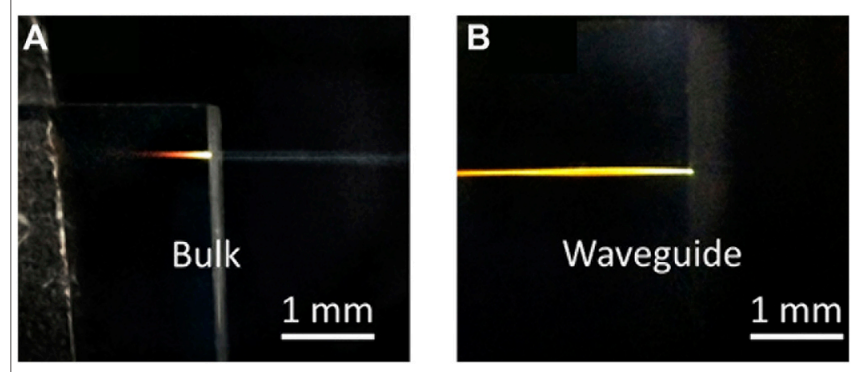

FIGURE 3 | The images of the spread of the light in (A) the bulk and (B) waveguide of the phosphate glass.

are adjusted when testing waveguide performance and guided-wave luminescence. For guidance performance, a linearly polarized $\mathrm{He}-\mathrm{Ne}$ laser at wavelength of $633 \mathrm{~nm}$ was utilized as the light source, as shown in Figure 2. Placing a half-wave plate behind the laser converted the linearly polarized direction. The incident linearly polarized light was into the waveguides via a $\times 20$ microscope objective lens. Another microscope objective lens was used to outcouple the light, and finally a CCD camera as a detector collected and imaged the light (a photodetector altered to measure the output power). Through the end coupling system, we study the propagation modes and propagation losses of the waveguide at $633 \mathrm{~nm}$. The propagation losses of the photonic structures were determined by directly measuring the light powers (by power meters) from the input and output end-facesThe propagation loss can be estimated with the following equation [33].

$$
\boldsymbol{P}_{\text {out }}=\boldsymbol{P}_{\text {in }}(1-\boldsymbol{R})^{2} \boldsymbol{e}^{-\alpha L} \boldsymbol{T}
$$

where $R$ is the Fresnel reflection coefficient. $L$ stands for the length of the waveguide. $T$ is related to the mismatch between the pump beam mode and waveguide mode. The value of mismatch is calculated by a numerical calculating software to be $~ 19$ and $23 \%$ for $30 \mathrm{~nm}$ - and $100 \mathrm{~nm}$-diameter waveguides, respectively (more details discussed in next section).

For guided-wave luminescence, a filter was inset the platform behind collected objective for removing optical

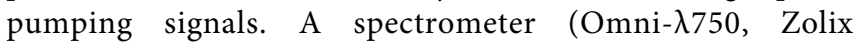
Instruments Co. Ltd.) was used as the detector in the platform for measuring the spectra of the up-conversion at room temperature.
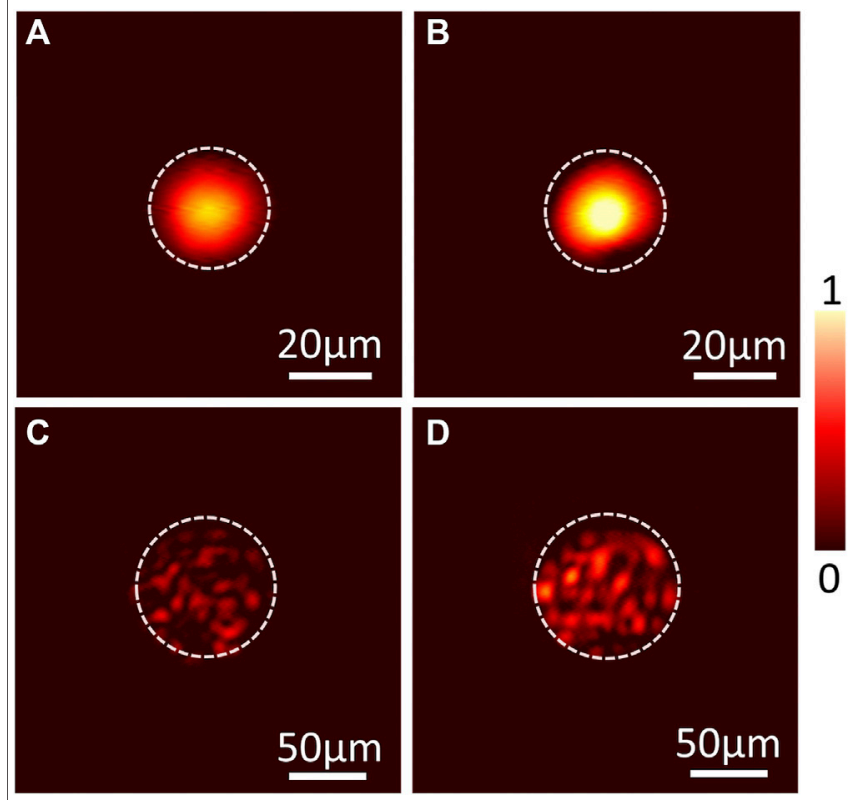

FIGURE 4 | The spatial modal profiles of the (A) TE and (B) TM modes for the phosphate glass cladding waveguide of $30 \mu \mathrm{m}$ diameter at a wavelength of $632.8 \mathrm{~nm}$; The spatial modal profiles of the (C) TE and (D) TM modes for the waveguide of $100 \mu \mathrm{m}$ diameter at $632.8 \mathrm{~nm}$. The dashed lines indicate the spatial locations of laser-induced tracks.

\section{RESULTS AND DISCUSSION}

Figures 3A, B shows the optical microscope images of the light entering the bulk and the waveguide of phosphate glass, respectively. The up-conversion luminescence light fast lost in the bulk interior. Therefore, a bright and short beam is observed at the interface of the bulk and fiber, which excited by $980 \mathrm{~nm}$ light pumping. On the contrary, the beam can be well propagated along the optical waveguide, and the light intensities are higher in the waveguide cores than in the bulk (the intensities can be detected in the waveguides, but not in the bulk). It means that the up-conversion luminescence light can be effectively limited in the waveguide written by the femtosecond laser.

Figure 4 depict the measured spatial modal profiles at the output of waveguide as the linearly polarized light at $633 \mathrm{~nm}$ is launched into the waveguides of 30 - and $100-\mu \mathrm{m}$-diameter. An additional linearly polarizer is inserted into the end-face coupling system (before the detector, not shown in Figure 2) to inspect the polarization features of the output beams. It is worthy to point out that 30 - and $100-\mu \mathrm{m}$-diameter waveguides could support arbitrary polarizations, and the mode distributions do not exhibit significant polarization dependence as a result of the symmetric morphology of the cladding structures. The TE (TM) modal profiles are demonstrated in Figures 4A,C (Figure 4B,D) with the diameters of 30 and $100 \mu \mathrm{m}$, respectively. Propagating beams supports near Gaussian mode and multi-mode excitation in 30 - and $100-\mu \mathrm{m}$-diameter waveguides. And the polarizations of the propagating beams are well maintained in the both core-size waveguides. 

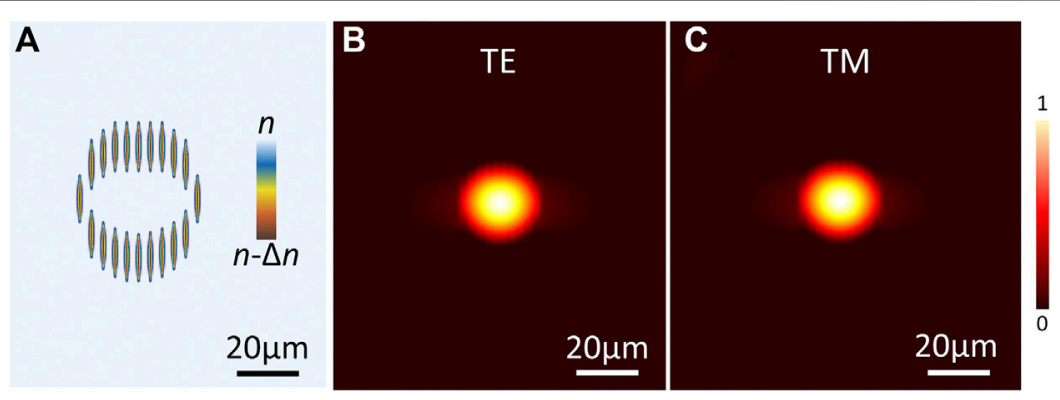

FIGURE 5 | (A) Re-constructure of refractive index distribution; (B,C) Simulated near-field modal profiles of the reconstructed distribution at TE and TM polarizations, respectively.

By assumption of a step-like refractive index profile and measuring the numerical aperture of the waveguides, we can estimate the maximum refractive index change of the waveguide core using the formula [34]:

$$
\Delta n \approx \frac{\sin ^{2} \theta_{m}}{2 n}
$$

where $\theta_{\mathrm{m}}$ is the maximum incident angular deflection at which no transmitted power change occurs. All the measurements were carried out at room temperature. $n=1.60$ is the refractive index of the bulk at $633 \mathrm{~nm}$ wavelength. By inserting a prism in the endface coupling system, the incident angle $(\theta)$ can be chaged. The maximum incident angular $\left(\theta_{\mathrm{m}}\right)$ can be confirmed to be $\sim 3.5^{\circ}$ with a difference of $0.3^{\circ}$ between both polarizations. In this work, we calculated maximum refractive index change $(\Delta n) \sim 1.2 \times 10^{-3}$. The refractive index at the end-face of $30-\mu \mathrm{m}$-diameter waveguide has been reconstructed (as shown in Figure 5A). The modal profiles are calculated by the FD-BPM algorithm (Rsoft ${ }^{\circledast}$ Beam PROP), which are shown in Figures 5B,C. The mismatch of the launched Gaussian beam $\left(E_{\mathrm{G}}\right)$ with modal profiles $\left(E_{\mathrm{P}}\right)$ of waveguides can be calculated by an overlap integral between both profiles. The mismatch coefficient $L$ can be expressed as [35]:

$$
T=\frac{1}{2}\left(\frac{\varepsilon_{0}}{\mu_{0}}\right) \int_{S} E_{G} \cdot E_{P} d S
$$

The calculated data are in good agreements with the experimental ones. It could be clearly seen that the two waveguides fabricated with the same parameters except size also exhibit the positively related refractive index alternations.

Based on the end-face coupling system at $633 \mathrm{~nm}$, we measured the light power of the input and output end-faces. The propagation losses were calculated to be $1.8 \pm 0.3 \mathrm{~dB} / \mathrm{cm}$ and $2.3 \pm 0.3 \mathrm{~dB} / \mathrm{cm}$ for 30 - and $100-\mu \mathrm{m}$-diameter waveguide. It indicates that, the larger the diameter of the waveguide brings the bigger the propagation loss due to some effects introduced by multi-mode, for instance mode-coupling. Consequently, the propagation losses can be reduced by optimizing the size of the optical waveguide (e.g., reducing the diameter of the waveguide) of the femtosecond laser writing $[36,37]$.
Up-conversion luminescence is well-known process, which converts near-infrared radiation (NIR) into visible light. An antiStokes process can convert two or more low energy photons into a higher energy photon. Here, a $980-n m$ continuous-wave laser was used to excite up-conversion luminescence through an end-face coupling system of optical fiber-waveguide physical contact end surfaces. As shown in Figure 6A, emission spectra were collected by a micro-objective output from the waveguides and detected by a spectrometer in the $400-700 \mathrm{~nm}$ range with a resolution of $0.1 \mathrm{~nm}$. The spectra of bulk and waveguide have two peak positions at 650 and $660 \mathrm{~nm}$, and the peak positions are almost the same. From 400 to $560 \mathrm{~nm}$, there is almost no difference between the emission spectra of bulk and waveguide. From $560 \mathrm{~nm}$, the emission spectrum of the waveguide began to increase significantly. The emission spectra are higher in the waveguides than in the bulk. For the up-conversion emissions, the intracavity light intensities from the waveguides are increased by $38.3 \%$ in comparison to the bulk. There is little difference between waveguides with diameters of 30 and $100 \mu \mathrm{m}$. The results show that the depressed cladding waveguide will be helpful for up-conversion applications in optical integrated devices.

Three possible up-conversion mechanisms for $\mathrm{Er}^{3+} / \mathrm{Yb}^{3+} \mathrm{co}-$ doped phosphate glasses are by excited state absorption (ESA), energy transfer (ET) and cross-relaxation (CR). Based on the energy match situation dependency on pumping power and the $\mathrm{Er}^{3+}$ and $\mathrm{Yb}^{3+}$ ion energy level diagram, the possible upconversion mechanism was discussed as known in Figure 6B. Under $980 \mathrm{~nm}$ excitation, the pumping laser operating excites electrons from the ground state to the $2 \mathrm{~F} 5 / 2$ excited state through ground state absorption (GSA) of the exciting photons, i.e.,4F7/2 $(\mathrm{Yb} 3+)+$ a photon $\rightarrow 2 \mathrm{~F} 5 / 2(\mathrm{Yb} 3+)$. After the first excitation to the $2 \mathrm{~F} 5 / 2$ level, a second photon is absorbed by the same ion (ESA), exciting it to $4 \mathrm{~F} 7 / 2$ state, i.e., $2 \mathrm{~F} 5 / 2(\mathrm{Yb} 3+)+$ a photon $\rightarrow$ 4 F7/2 (Er3+). For luminescence, there may be two attenuation possibilities: 1) electrons return from the $4 \mathrm{~F} 7 / 2$ state $(\mathrm{Yb} 3+)$ or $4 \mathrm{I} 15 / 2$ state $(\mathrm{Er} 3+)$ to the ground state by releasing energy; 2$)$ the rapid nonradiative decay from $4 \mathrm{~F} 7 / 2-4 \mathrm{~F} 9 / 2,4 \mathrm{~S} 3 / 2$ and $2 \mathrm{H} 11 / 2$ level results in the dominant population at $4 \mathrm{~F} 9 / 2,4 \mathrm{~S} 3 / 2$ and $2 \mathrm{H} 11 / 2$ states. Further electrons at excited levels return to ground level and emit photons. 

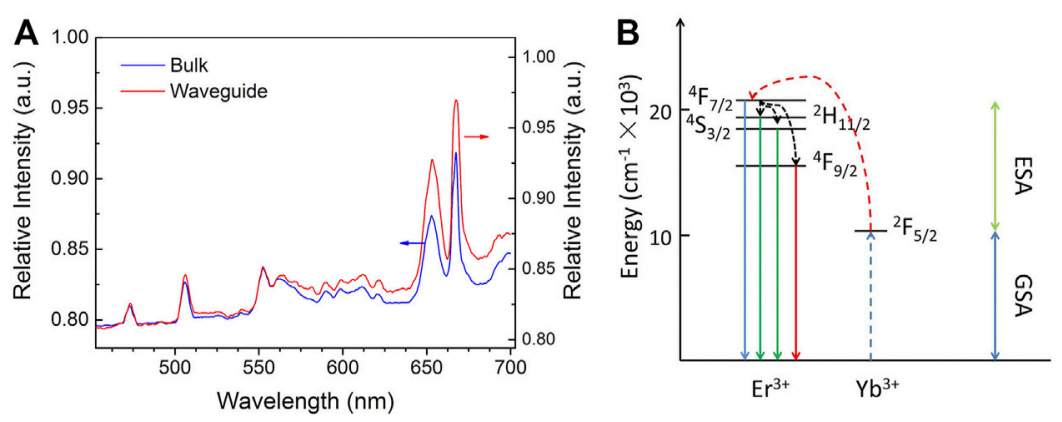

FIGURE 6 | (A) Up-conversion emission spectra detected in the bulk material and waveguides. (B) Diagram of the up-conversion emission under 980 nm excitation.

Compared with the previous phosphate glass optical waveguide, the depressed waveguides prepared by femtosecond laser writing have lower losses [38-40]. The waveguides can support optical transmission in two polarization directions of TE and TM. It can maintain the excellent up-conversion performance of the material in the waveguide core [41]. The waveguide can be designed for a wider range of cross-sectional dimensions.

\section{CONCLUSION}

We have successfully fabricated the waveguides of different diameters in the $\mathrm{Er}, \mathrm{Yb}$ co-doped phosphate glass by direct femtosecond laser writing. The measured modal profiles of waveguides exhibit fundamental transmission at $633 \mathrm{~nm}$ wavelengths. Propagation loss as low as $1.8 \mathrm{~dB} / \mathrm{cm}$ is obtained. The near fundamental mode and multi-mode are supported via controlling the diameter of waveguide. The up-conversion emissions of the waveguides have been obtained under excitation of $980 \mathrm{~nm}$ radiations. We observed the emission spectrum and found that the depressed cladding waveguide will be helpful for up-conversion applications in optical integrated devices. These cladding waveguides show good

\section{REFERENCES}

1. Haase M, Schäfer H. Upconverting Nanoparticles. Angew Chem Int Ed (2011) 50:5808-29. doi:10.1002/anie.201005159

2. Auzel F. Upconversion and Anti-Stokes Processes with F and D Ions in Solids. Chem Rev (2004) 104:139-74. doi:10.1021/cr020357g

3. Xu R, Tian Y, Hu L, Zhang J. Enhanced Emission of $27 \mu \mathrm{m}$ Pumped by Laser Diode from Er3+/Pr3+-Codoped Germanate Glasses. Opt Lett (2011) 36: 1173-5. doi:10.1364/OL.36.001173

4. Zhang G, Zhou Q, Yu C, Hu L, Chen D. Neodymium-Doped Phosphate Fiber Lasers with an All-Solid Microstructured Inner Cladding. Opt Lett (2012) 37: 2259-61. doi:10.1364/ol.37.002259

5. Bernier M, Fortin V, El-Amraoui M, Messaddeq Y, Vallée R. $377 \mu \mathrm{m}$ Fiber Laser Based on Cascaded Raman Gain in a Chalcogenide Glass Fiber. Opt Lett (2014) 39:2052-5. doi:10.1364/ol.39.002052

6. Basavapoornima C, Maheswari T, Depuru SR, Jayasankar CK. Sensitizing Effect of $\mathrm{Yb} 3+$ Ions on Photoluminescence Properties of Er3+ Ions in lead potential for integrated optical circuits and miniature visible fluorescence waveguide devices.

\section{DATA AVAILABILITY STATEMENT}

The raw data supporting the conclusion of this article will be made available by the authors, without undue reservation.

\section{AUTHOR CONTRIBUTIONS}

YZ performed simulation, sorted out the data and completed the paper writing. CC performed waveguide fabrication and test. $\mathrm{LH}$ made the phosphate glass sample. FC supervised the program and gave some suggestions to this paper.

\section{FUNDING}

This research was funded by the National Natural Science Foundation of China (No. 11904212), China Postdoctoral Science Foundation (No. 2019M662455).

Phosphate Glasses: Optical Fiber Amplifiers. Opt Mater (2018) 86:256-69. doi:10.1016/j.optmat.2018.09.027

7. Wang P, Lu M, Gao F, Guo H, Xu Y, Hou C, et al. Luminescence in the Fluoride-Containing Phosphate-Based Glasses: A Possible Origin of Their High Resistance to Nanosecond Pulse Laser-Induced Damage. Sci Rep (2015) 5:8593. doi: $10.1038 /$ srep 08593

8. Dwivedi Y, Mishra K, Rai SB. Synthesis of Bright Multicolor Down and Upconversion Emitting Y2Te4O11:Er:Yb Nanocrystals. J Alloys Comp (2013) 572:90-6. doi:10.1016/j.jallcom.2013.03.252

9. Yang X, Fu Z, Yang Y, Zhang C, Wu Z, Sheng T. Optical Temperature Sensing Behavior of High-Efficiency Upconversion: Er 3+ - Yb 3+ Co-Doped $\mathrm{NaY}(\mathrm{MoO} 4$ ) 2 Phosphor. J Am Ceram Soc (2015) 98:2595-600. doi:10.1111/jace.13624

10. Dong H, Sun L-D, Yan C-H. Lanthanide-Doped Upconversion Nanoparticles for Super-Resolution Microscopy. Front Chem (2020) 8:619377. doi:10.3389/ fchem.2020.619377

11. Tan Y, Hu X, Liu M, Liu X, Lv X, Li Z, et al. Simultaneous Visualization and Quantitation of Multiple Steroid Hormones Based on Signal-Amplified 
Biosensing with Duplex Molecular Recognition. Chem Eur J (2017) 23: 10683-9. doi:10.1002/chem.201702220

12. Chen S, Song W, Cao J, Hu F, Guo H. Highly Sensitive Optical Thermometer Based on FIR Technique of Transparent NaY2F7:Tm3+/Yb3+ Glass Ceramic. J Alloys Comp (2020) 825:154011. doi:10.1016/j.jallcom.2020.154011

13. Zhang Y, Li M, Li J, Tang J, Cao W, Wu Z. Optical Properties of Er3+/Yb3+ Co-Doped Phosphate Glass System for NIR Lasers and Fiber Amplifiers. Ceramics Int (2018) 44:22467-72. doi:10.1016/j.ceramint.2018.09.015

14. Shen LF, Chen BJ, Lin H, Pun EYB. Praseodymium Ion Doped Phosphate Glasses for Integrated Broadband Ion-Exchanged Waveguide Amplifier. J Alloys Comp (2015) 622:1093-7. doi:10.1016/j.jallcom.2014.11.033

15. Jia Y, He R, Vázquez de Aldana JR, Liu H, Chen F. Femtosecond Laser Direct Writing of Few-Mode Depressed-Cladding Waveguide Lasers. Opt Express (2019) 27:30941-51. doi:10.1364/oe.27.030941

16. He S, Yang Q, Zhang B, Ren Y, Liu H, Wu P, et al. A Waveguide Mode Modulator Based on Femtosecond Laser Direct Writing in KTN Crystals. Results Phys (2020) 18:103307. doi:10.1016/j.rinp.2020.103307

17. Giallorenzi TG, West EJ, Kirk R, Ginther R, Andrews RA. Optical Waveguides Formed by Thermal Migration of Ions in Glass. Appl Opt (1973) 12:1240-5. doi:10.1364/ao.12.001240

18. Ohya S, Chiaro B, Megrant A, Neill C, Barends R, Chen Y, et al. Room Temperature Deposition of Sputtered TiN Films for Superconducting Coplanar Waveguide Resonators. Supercond Sci Technol (2013) 27:015009. doi:10.1088/0953-2048/27/1/015009

19. Duan Y, Dekker P, Ams M, Palmer G, Withford MJ. Time Dependent Study of Femtosecond Laser Written Waveguide Lasers in Yb-Doped Silicate and Phosphate Glass. Opt Mater Express (2015) 5:416-22. doi:10.1364/ ome.5.000416

20. Davis KM, Miura K, Sugimoto N, Hirao K. Writing Waveguides in Glass with a Femtosecond Laser. Opt Lett (1996) 21:1729-31. doi:10.1364/ol.21.001729

21. Tan D, Sharafudeen KN, Yue Y, Qiu J. Femtosecond Laser Induced Phenomena in Transparent Solid Materials: Fundamentals and Applications. Prog Mater Sci (2016) 76:154-228. doi:10.1016/ j.pmatsci.2015.09.002

22. Chen Q, Fang Z, Song H, Zhang F, Nie J, Chen Z, et al. Femtosecond Laser Induced Space-Selective Precipitation of $\mathrm{Cr} 3+$-Doped $\mathrm{ZnAl} 2 \mathrm{O} 4$ crystal in Glass. J Alloys Comp (2017) 699:243-6. doi:10.1016/j.jallcom.2016.12.332

23. Yao H, Zaiter R, Cavillon M, Sapaly B, Calzavara F, Delullier P, et al. Photosensitivity of Barium Germano-Gallate Glasses under Femtosecond Laser Direct Writing for Mid-IR Applications. Ceramics Int (2021) 47(24): 34235-41. doi:10.1016/j.ceramint.2021.08.333

24. Macias-Montero M, Muñoz F, Sotillo B, del Hoyo J, Ariza R, Fernandez P, et al. Femtosecond Laser Induced Thermophoretic Writing of Waveguides in Silicate Glass. Sci Rep (2021) 11(1):1-12. doi:10.1038/s41598-021-87765-z

25. Michele VD, Royon M, Marin E, Alessi A, Morana A, Boukenter A, et al. NearIR- and UV-Femtosecond Laser Waveguide Inscription in Silica Glasses. Opt Mater Express (2019) 9(12):4624-33. doi:10.1364/OME.9.004624

26. Liu Z, Liao Y, Wang Z, Zhang Z, Liu Z, Qiao L, et al. Fabrication of an Optical Waveguide-Mode-Field Compressor in Glass Using a Femtosecond Laser. Materials (2018) 11(10):1926. doi:10.3390/ma11101926

27. Lancaster DG, Li Y, Duan Y, Gross S, Withford MW, Monro TM. Er3+Active $\mathrm{Yb} 3+\mathrm{Ce} 3+\mathrm{Co}$-Doped Fluorozirconate Guided-Wave Chip Lasers. IEEE Photon Technol Lett (2016) 28(21):2315-8. doi:10.1109/LPT.2016.2592514

28. Smayev MP, Dorofeev VV, Moiseev AN, Okhrimchuk AG. Femtosecond Laser Writing of a Depressed Cladding Single Mode Channel Waveguide in HighPurity Tellurite Glass. J Non-Crystalline Sol (2018) 480:100-6. doi:10.1016/ j.jnoncrysol.2017.11.007

29. Lancaster DG, Gross S, Ebendorff-Heidepriem H, Withford MJ, Monro TM, Jackson SD. Efficient $29 \mu \mathrm{m}$ Fluorozirconate Glass Waveguide Chip Laser. Opt Lett (2013) 38(14):2588-91. doi:10.1364/OL.38.002588

30. Okhrimchuk AG, Pryamikov AD, Gladyshev AV, Alagashev GK, Smayev MP, Likhov VV, et al. Direct Laser Written Waveguide in Tellurite Glass for
Supercontinuum Generation in $2 \mu \mathrm{m}$ Spectral Range. J Lightwave Technol (2020) 38(6):1492-500. doi:10.1109/JLT.2019.2954862

31. Santos SNC, Almeida JMP, Almeida GFB, Mastelaro VR, Mendonca CR. Fabrication of Waveguides by Fs-Laser Micromachining in Dy3+/Eu3+Doped Barium Borate Glass with Broad Emission in the Visible Spectrum. Opt Commun (2018) 427:33-6. doi:10.1016/j.optcom.2018.06.026

32. Khalid M, Chen GY, Ebendorff-Heidepreim H, Lancaster DG. Femtosecond Laser Induced Low Propagation Loss Waveguides in a Lead-Germanate Glass for Efficient Lasing in Near to Mid-IR. Sci Rep (2021) 11:10742. doi:10.1038/ s41598-021-90249-9

33. Zhang L, Guo T, Ren Y, Cai Y, Mackenzie MD, Kar AK, et al. Cooperative UpConverted Luminescence in Yb,Na:CaF2 Cladding Waveguides by Femtosecond Laser Inscription. Opt Commun (2019) 441:8-13. doi:10.1016/j.optcom.2019.01.032

34. Siebenmorgen J, Petermann K, Huber G, Rademaker K, Nolte S, Tünnermann A. Femtosecond Laser Written Stress-Induced Nd:Y3Al5O12 (Nd:YAG) Channel Waveguide Laser. Appl Phys B (2009) 97:251-5. doi:10.1007/ s00340-009-3697-3

35. Cheng C, Romero C, de Aldana JRV, Chen F. Superficial Waveguide Splitters Fabricated by Femtosecond Laser Writing of LiTaO 3 crystal. Opt Eng (2015) 54(6):067113. doi:10.1117/1.OE.54.6.067113

36. Manzani D, Montesso M, Mathias CF, Krishanaiah KV, Ribeiro SJL, Nalin M. Visible Up-Conversion and Near-Infrared Luminescence of Er3+/Yb3+ CoDoped SbPO4-GeO2 Glasses. Opt Mater (2016) 57:71-8. doi:10.1016/ j.optmat.2016.04.019

37. Chen F, de Aldana JRV. Optical Waveguides in Crystalline Dielectric Materials Produced by Femtosecond-Laser Micromachining. Laser Photon Rev (2014) 8(2):251-75. doi:10.1002/lpor.201300025

38. Liu C-X, Pan H, Lv J-Y, Chen J-Y, Lin S-B, Zheng R-L, et al. An Infrared 2D Nd3+-Doped Phosphate Glass Waveguide Formed by Proton Implantation and Femtosecond Laser Ablation. Infrared Phys Tech (2021) 113:103578. doi:10.1016/j.infrared.2020.103578

39. Taccheo S, Della Valle G, Osellame R, Cerullo G, Chiodo N, Laporta P, et al. Er: Yb-Doped Waveguide Laser Fabricated by Femtosecond Laser Pulses. Opt Lett (2004) 29(22):2626-8. doi:10.1364/OL.29.002626

40. Fletcher LB, Witcher JJ, Troy N, Reis ST, Brow RK, Krol DM. Direct Femtosecond Laser Waveguide Writing Inside Zinc Phosphate Glass. Opt Express (2011) 19(9):7929-36. doi:10.1364/OE.19.007929

41. Liu H, Jia Y, Vázquez de Aldana JR, Jaque D, Chen F. Femtosecond Laser Inscribed Cladding Waveguides in Nd:YAG Ceramics: Fabrication, Fluorescence Imaging and Laser Performance. Opt Express (2012) 20(17): 18620-9. doi:10.1364/OE.20.018620

Conflict of Interest: The authors declare that the research was conducted in the absence of any commercial or financial relationships that could be construed as a potential conflict of interest.

The handling editor declared a past co-authorship with one of the authors FC.

Publisher's Note: All claims expressed in this article are solely those of the authors and do not necessarily represent those of their affiliated organizations, or those of the publisher, the editors and the reviewers. Any product that may be evaluated in this article, or claim that may be made by its manufacturer, is not guaranteed or endorsed by the publisher.

Copyright (๑) 2022 Zhou, Cheng, Hu and Chen. This is an open-access article distributed under the terms of the Creative Commons Attribution License (CC BY). The use, distribution or reproduction in other forums is permitted, provided the original author(s) and the copyright owner(s) are credited and that the original publication in this journal is cited, in accordance with accepted academic practice. No use, distribution or reproduction is permitted which does not comply with these terms. 